\title{
Shared decision making in psychiatric practice and the primary care setting is unique, as measured using a 9-item Shared Decision Making Questionnaire (SDM-Q-9)
}

\author{
This article was published in the following Dove Press journal: \\ Neuropsychiatric Disease and Treatment \\ 30 July 2013 \\ Number of times this article has been viewed
}

\section{Carlos De las Cuevas 1,2 \\ Wenceslao Peñate ${ }^{3}$ \\ Lilisbeth Perestelo-Pérez ${ }^{2,4}$ \\ Pedro Serrano-Aguilar ${ }^{2,4}$ \\ 'Department of Psychiatry, University of La Laguna, Tenerife, Spain; ${ }^{2}$ Health Services Research Network for Chronic Diseases (REDISSEC), Tenerife, Spain; ${ }^{3}$ Department of Personality, Assessment and Psychological Treatments, University of La Laguna, Tenerife, Spain; \\ ${ }^{4}$ Evaluation Unit, Canary Island Health Service, Santa Cruz de Tenerife, Spain}

Correspondence: Carlos De las Cuevas Department of Psychiatry, University of La Laguna, School of Medicine, Campus de Ofra s/n, 3807I San Cristóbal de La Laguna, Canary Islands, Spain Tel +3460952 I405

Fax +3492 23I 9353

Email cdelascuevas@gmail.com
Background: To measure and compare the extent to which shared a decision making (SDM) process is implemented both in psychiatric outpatient clinical encounters and in the primary care setting from the patient's perspective.

Methods: A total of 1,477 patients recruited from the Canary Islands Health Service mental health and primary care departments were invited to complete the nine-item Shared Decision Making Questionnaire (SDM-Q-9) immediately after their consultation. MANCOVA, Student's $t$-test, and Pearson correlations were used to assess the relationship and differences between SDM-Q-9 scores in patient samples.

Results: No differences were found in SDM-Q-9 total scores between the two patient samples, but there were relevant differences when item by item analysis was applied; differences were observed according to the different steps of the SDM process. SDM is present to a very limited extent in the routine psychiatric setting compared to primary care. Patients' age, education, type of appointment, and treatment decision all play a specific role in predicting SDM.

Conclusion: The study provides evidence that SDM is a complex process that needs to be analyzed according to its different steps. SDM patterns were different in the primary care and psychiatric outpatient care settings and reflect quite a different perspective of the decision making process.

Keywords: primary care patients, psychiatric outpatients, SDM-Q-9, shared decision making

\section{Background}

Choice, self-determination, and empowerment are foundational values for every patient, especially for those with psychiatric disorders. ${ }^{1}$ Shared decision making (SDM) is a clinical model that endorses these values and implements the basic rights of a group of patients who have not sufficiently benefited from consumer empowerment in other medical fields. ${ }^{2}$ SDM is an alternative to both the "paternalistic model" in which the physician unilaterally renders an opinion from above, and "pure consumerism", which reduces the physician to a dispassionate technocrat dispensing information concerning statistical risks and benefits of alternate treatment approaches. The model embraces the inherent vulnerability of the patient facing serious illness and who must make decisions that carry serious consequences. ${ }^{3}$

SDM is an interactive process of the clinical decision making model that ensures that both patient and physician are equally and actively involved and share information in order to come to an agreement, for which they are jointly responsible. ${ }^{4}$ 
The essential components of the SDM model include health professional and patient participation at all steps of the decision making process; these steps include professional disclosure, exchange of information, deliberation about options, patients' preferences, negotiation, and coming to an agreement on a decision to implement. ${ }^{5}$ In this sense, SDM is a sequential hierarchic process where each step makes sense by itself and can take place independently of other steps expressing specific communication patterns. ${ }^{6}$ Consequently, the patient-professional relationship can vary in SDM as a whole and throughout each step of the process, with each step potentially including distinct forms of SDM.

Although there have not been a large number of SDM studies in the mental health field to date, the positive effects of SDM are comparable to those documented in general non-mental health patient groups, suggesting that future research could confirm the usefulness of SDM for patients with psychiatric disorders. ${ }^{7}$ Nevertheless, the positive impact of SDM has been shown in improving patient treatment compliance, knowledge, and satisfaction, activation of and increasing involvement in decision making, fewer psychiatric symptoms, and an overall trend towards a decline in hospitalization. ${ }^{7-11}$ Given the considerable differences between the two therapeutic settings, it appears logical to suspect that despite the positive effects recorded in the mental health care setting, major differences could exist when comparing with SDM in general non-mental health patients at different steps of the SDM process.

Moreover, not all patients are prepared, suitable, or want to participate to the same degree in the decision-making process about the treatment of their disease. The suitability of a decision in SDM depends on several variables: (1) the characteristics of medical care, such as specialized or primary care; (2) socio-demographic variables such as age or education level; (3) clinical context, such as type of appointment or treatment decision; and (4) personal variables, such as patient preferences or the responsibility of healthcare professionals. ${ }^{12}$

According to the latter personal variables, previous research in our health care setting has revealed a positive attitude towards SDM both in mental health professionals ${ }^{13}$ and among psychiatric outpatients. ${ }^{14,15}$ However, further studies are needed to address the extent and characteristics to which this apparently accepted model is reflected in different medical care, the daily practice of mental health professionals, and the influence of clinical context in the process.

The aim of this study was to measure and compare the SDM process both in psychiatric clinical encounters and in the primary care setting from the patient's perspective. The comparison took into account not only the final SDM consideration, but also the unique steps involved in the SDM process. To achieve this goal we used the nine-item Shared Decision Making Questionnaire (SDM-Q-9), a theory-driven self-report questionnaire that assess the SDM process in clinical encounters from the patient perspective; we also worked to clarify underlying concepts by defining theoretical key features and nine practical steps. ${ }^{16}$

It was expected that the two different medical care settings (primary care and psychiatric outpatient care) would also differ as to how they proceed in the different steps of the SDM process. We also sought to understand how clinical and sociodemographic variables influence the interactive processes of the clinical decision making model.

\section{Methods}

\section{Sample recruitment}

A total of 1,477 consecutive patients were recruited from one Community Mental Health Center (682 psychiatric outpatients seen by five psychiatrists) and from two primary care centers (795 primary care patients seen by 20 general practitioners). These patients belonged to a basic health care population of 135,000 inhabitants using the Canary Islands Health Service. Primary care patients being treated by mental health facilities were excluded from the study.

Each patient completed an anonymous questionnaire that included the patient's sociodemographic variables (sex, age, educational level), clinical variables (appointment type, selfreported diagnosis, treatment decision), health professional variables (doctor, sex, age), and the SDM-Q-9, completed immediately after the patient's clinical consultation with their psychiatrist/general practitioner and not in the presence of the treating health professional.

Each participant received a full written explanation of the study, after which they signed an informed consent document approved by the local ethics committee. To rate the SDM-Q-9, participants were instructed to think about their last consultation and to use this consultation as a reference for the rating. Questionnaires were self-administered in all cases. Patients did not receive any financial compensation for their participation. The study was performed from September to December 2012.

We considered two appointment types: New and followup scheduled appointments correspond to patients who have any medical or psychiatric conditions that should be scheduled for planned follow-up visits at periodic intervals, both in order to monitor their condition and to modify treatment 
if needed (including prescription refills); non-scheduled consultations involve patients seeking non-scheduled medical or psychiatric care in situations they perceive as worsening their condition or in the event of an emergency, but which may in fact often not be a true emergency.

Treatment decision options were categorized as follows: prescription of a new treatment; maintenance of the previous treatment; and modification of previous treatment by increasing or decreasing dosages of the drug treatment.

The study was performed in accordance with the Code of Ethics of the Declaration of Helsinki and all procedures and consent forms were reviewed and approved by the Ethics Committee of the University Hospital Nuestra Señora de la Candelaria.

\section{Instrument}

The SDM-Q-9 is a valid, reliable, and brief self-report questionnaire that assesses the patient' view of the decision making process in a consultation; ${ }^{17}$ it has been adapted and validated into Spanish by De las Cuevas et al. ${ }^{18}$ The questionnaire consists of nine items, each describing one step of the SDM process. ${ }^{16}$ The questionnaire was developed to show the extent to which patients feel they were involved in the process by scoring nine items from 0 to 5 on a six-point Likert scale; the scale ranges from "completely disagree" (0) to "completely agree" (5). Summing up all items leads to a raw total score between 0 and 45. Multiplication of the raw score by 20/9 provides a score forced (transformed) to range from 0 to 100 , where 0 indicates the lowest possible level of SDM and 100 indicates the highest extent of SDM. As it is more intuitively interpretable, the original authors encourage use of the transformed score. Table 1 shows the SDM-Q-9 items, their contents, and the steps involved in the SDM process.

\section{Statistical analyses}

The samples' demographic information was reported by frequency analyses. Demographic information was also collected for patients who were invited but who refused to take part. To estimate differences in SDM perceptions (item by item and total score) between primary care patients and psychiatric patients, Student's $t$-tests were performed. Effect sizes were calculated by Cohen's statistic. In order to analyze the orientation of item by item scores, direct scores were transformed into two categories, "disagree" (responses from 0 to 2), and "agree" (responses from 3 to 5). Finally, regression analyses were performed to estimate the best predictors of SDM, both total score and item by item. To identify only those variables that play a relevant role in the prediction of SDM scores, a step-by-step model was used. This model only introduces into the equation those variables with a significant contribution.

\section{Results \\ Samples}

Table 2 shows the sample distribution according to age, sex, educational level, and self-reported diagnoses. Six hundred and eighty-two consecutive psychiatric outpatients were invited to take part, and 571 (83.7\%) accepted. Nonparticipants were slightly older than participants $(50.4 \pm 13.7$ versus $49.5 \pm 16.4$ years, respectively; no statistically significant differences) and included more men (43.1\% versus $35.1 \%$;

Table I Items and contents of the SDM-Q-916 and steps in the SDM process ${ }^{17}$

\begin{tabular}{|c|c|c|}
\hline SDM-Q-9 Items & Contents & Steps in the SDM Process \\
\hline $\begin{array}{l}\text { I. My doctor made clear that a decision needs } \\
\text { to be made }\end{array}$ & $\begin{array}{l}\text { Recognizing that a decision needs } \\
\text { to be made }\end{array}$ & $\begin{array}{l}\text { Disclosure that a decision needs } \\
\text { to be made }\end{array}$ \\
\hline $\begin{array}{l}\text { 2. My doctor wanted to know exactly how I want } \\
\text { to be involved in making the decision }\end{array}$ & $\begin{array}{l}\text { Asking for preferred involvement } \\
\text { in decision-making }\end{array}$ & Formulation of equality of partners \\
\hline $\begin{array}{l}\text { 3. My doctor told me that there are different } \\
\text { options for treating my condition }\end{array}$ & $\begin{array}{l}\text { Informing that different options are } \\
\text { available }\end{array}$ & Equipoise statement \\
\hline $\begin{array}{l}\text { 4. My doctor precisely explained the advantages } \\
\text { and disadvantages of the treatment options }\end{array}$ & $\begin{array}{l}\text { Explaining the advantages and } \\
\text { disadvantages of the options }\end{array}$ & $\begin{array}{l}\text { Informing patient of the benefits } \\
\text { and risks of the options }\end{array}$ \\
\hline 5. My doctor helped me understand all the information & Helping to understand the information & $\begin{array}{l}\text { Investigation of the patient's } \\
\text { understanding and expectations }\end{array}$ \\
\hline 6. My doctor asked me which treatment option I prefer & Asking for preferred option & Identification of preferences \\
\hline $\begin{array}{l}\text { 7. My doctor and I weighed the different treatment } \\
\text { options thoroughly }\end{array}$ & Weighing the options (doctor and patient) & Negotiation \\
\hline 8. My doctor and I selected a treatment option together & Selecting an option (doctor and patient) & Shared decision \\
\hline $\begin{array}{l}\text { 9. My doctor and I came to an agreement on } \\
\text { how to proceed }\end{array}$ & $\begin{array}{l}\text { Agreeing on how to proceed (doctor and } \\
\text { patient) }\end{array}$ & Arrangement of follow-up \\
\hline
\end{tabular}

Abbreviations: SDM-Q-9, nine-item Shared Decision-Making Questionnaire; SDM, Shared Decision-Making. 
Table 2 Sample distribution according to sex, age, educational level, and self-reported diagnoses

\begin{tabular}{|c|c|c|c|c|}
\hline & \multicolumn{2}{|c|}{ Psychiatric outpatients } & \multicolumn{2}{|l|}{ Primary care patients } \\
\hline Participation rate & $571 / 682=83.7 \%$ & & $540 / 795=67.9 \%$ & \\
\hline \multicolumn{5}{|l|}{ Age } \\
\hline Mean age $\pm S D$ & $49.5 \pm 16.4$ years & & $44.7 \pm 16.4$ years & \\
\hline Rank & 18-86 years & & I8-87 years & \\
\hline \multicolumn{5}{|l|}{ Gender } \\
\hline Male & $35.1 \%$ & & $30.0 \%$ & \\
\hline Female & $64.9 \%$ & & $70.0 \%$ & \\
\hline \multicolumn{5}{|l|}{ Educational level } \\
\hline No formal education & $13.1 \%$ & & $11.9 \%$ & \\
\hline Primary & $41.3 \%$ & & $40.9 \%$ & \\
\hline Secondary & $34.0 \%$ & & $33.7 \%$ & \\
\hline University & $11.5 \%$ & & $13.5 \%$ & \\
\hline \multicolumn{5}{|l|}{ Self-reported diagnoses } \\
\hline Psychiatric outpatients $90 \%$ & Depressive disorder & $35 \%$ & Respiratory problems & $18 \%$ \\
\hline \multirow[t]{5}{*}{ Primary care patients $74 \%$} & Anxiety disorder & $23 \%$ & Unspecified problems & $16 \%$ \\
\hline & Schizophrenia & $19 \%$ & Cardiovascular problems & $15 \%$ \\
\hline & Bipolar disorder & $6 \%$ & Digestive problems & $12 \%$ \\
\hline & Personality disorder & $2 \%$ & Endocrine, metabolic and nutritional & $8 \%$ \\
\hline & Other diagnoses & $5 \%$ & Psychosocial problem & $5 \%$ \\
\hline
\end{tabular}

Abbreviation: SD, standard deviation.

$\left.\chi^{2}=2.43, P=0.073\right)$. Seven hundred and ninety-five primary care patients were invited to take part, and $540(67.9 \%)$ accepted. Nonparticipants were significantly older than participants $(47.6 \pm 15.8$ versus $44.7 \pm 16.4$ respectively; $t=2.32, P=0.02)$ and included more men ( $40 \%$ versus $\left.29.8 \% ; \chi^{2}=7.66, P=0.006\right)$.

\section{Descriptive analyses of the SDM-Q-9 items}

An initial analysis was performed to compare SDM-Q-9 total score between primary care patients and psychiatric outpatients. Student's $t$-test for total score (0 to 100 transformed) did not reveal any statistically significant differences between them (primary care patients: $67.5 \pm 21.9$; psychiatric outpatients: $68.4 \pm 19.7 ; t[1109]=-0.7)$. Since there were no statistically significant differences, new $t$-tests were performed, but taking into account the different items (representing different contents/steps in SDM). Table 3 summarizes the data obtained.

As can be observed, since no disparity was found for total score, specific items showed statistically significant differences between the two groups of patients. The exception was item 8 , and this absence of differences is coherent with the absence of differences for total score because the item's content refers to the final patient/professional shared decision.

The pattern of differences observed revealed that psychiatric patient score is higher in initial items (when

Table 3 Mean differences between primary care patients and psychiatric outpatients for the different steps in the SDM process, according to SDM-Q-9 items

\begin{tabular}{|c|c|c|c|c|c|c|c|}
\hline \multirow[t]{2}{*}{$\begin{array}{l}\text { Shared decision-making process steps } \\
\text { corresponding to SDM-Q-9 Items }\end{array}$} & \multicolumn{2}{|c|}{$\begin{array}{l}\text { Primary care } \\
\text { patients }(n=540)\end{array}$} & \multicolumn{2}{|c|}{$\begin{array}{l}\text { Psychiatric out- } \\
\text { patients }(n=57 I)\end{array}$} & \multirow[t]{2}{*}{$\mathbf{z}$} & \multirow[t]{2}{*}{$P$} & \multirow[t]{2}{*}{ d } \\
\hline & $M$ & SD & $M$ & SD & & & \\
\hline Disclosure that a decision needs to be made & 3.66 & 1.34 & 4.37 & 0.90 & -9.51 & 0.000 & 0.62 \\
\hline Formulation of equality of partners & 3.07 & 1.55 & 4.19 & 1.06 & -12.99 & 0.000 & 0.84 \\
\hline Equipoise statement & 3.56 & 1.45 & 2.86 & 1.47 & -8.08 & 0.000 & 0.48 \\
\hline Informing on the options' benefits and risks & 3.60 & $\mathrm{I} .43$ & 2.89 & 1.48 & -8.22 & 0.000 & 0.49 \\
\hline $\begin{array}{l}\text { Investigation of patient's understanding and } \\
\text { expectations }\end{array}$ & 4.24 & 1.14 & 4.09 & 1.05 & -3.77 & 0.020 & 0.14 \\
\hline Identification of preferences & 3.07 & 1.65 & 2.61 & 1.46 & -5.38 & 0.000 & 0.30 \\
\hline Negotiation & 3.09 & 1.54 & 2.75 & 1.46 & -4.39 & 0.000 & 0.23 \\
\hline Shared decision & 2.71 & 1.80 & 2.69 & 1.48 & -0.60 & 0.546 & 0.01 \\
\hline Arrangement of follow-up & 3.37 & 1.68 & 4.33 & 0.88 & -9.94 & 0.000 & 0.72 \\
\hline
\end{tabular}

Abbreviations: SDM, Shared Decision-Making; SDM-Q-9, nine-item Shared Decision-Making Questionnaire; $d$, Cohen's statistic; M, median; $P$, probability; SD, standard deviation; z, z scores from Mann-Whitney's $U$. 
professionals notify their patients about the need to take a decision, and how patients are ready to take part). Primary care patients score is higher for the intermediate items (options, patients' preferences, negotiation, etc). For the final item (follow-up), psychiatric patients once again obtained higher scores. According to the effect sizes, greater effects were obtained when psychiatric patients scored higher (especially in "formulation of equality of partners"), moving from medium to high effect coefficients. Item 5 (patient's expectation) did not attain statistical significance.

A second analysis was performed to determine the orientation of such differences, ie, the level of agreement of both groups of patients with the different contents of SDM. As was noted above, every item was summarized into two categories, "disagree" (responses from 0 to 2 ) and "agree" (responses from 3 to 5). For better understanding, the results were converted to percentages. Table 4 summarizes the data obtained.

Although the transformed scores of the questionnaire recorded in both samples were almost identical (68.38 \pm 19.7 for psychiatric outpatients and $67.50 \pm 21.9$ for primary care patients), the results obtained item by item were once again very different, reflecting quite a different perspective of the decision making process in their consultations carried out (Table 4). While most primary care patients agreed with each and every one of the questionnaire items, most psychiatric outpatients disagreed with five of the items - in short, primary care patients scored higher.

Among psychiatric outpatients, most agree with their initial psychiatrist disclosure that a decision needs to be made and that their doctor wanted to know exactly how they want to be involved in making the decision. However, they in large part believed that they were not informed about the different options available or the advantages and disadvantages of said options. Most reported that their psychiatrist helped them understand the information, but that they were not asked about which treatment option they preferred, that there was no negotiation, that the selection of the treatment was not a consensus decision, and finally that an arrangement for follow-up was made.

Taking into account the different profiles between primary care and psychiatric care in SDM, the next analysis sought to ascertain whether contextual clinical variables such as appointment type (follow-up scheduled appointments and non-scheduled consultations), treatment decisions (maintenance of the previous treatment, modification, and new treatment), and sociodemographic variables (age, sex, and educational level) played a significant role in predicting those profiles. Several regression analyses were performed for items, as dependent variables. Contextual and sociodemographic variables, as well as sample type (primary care and psychiatry care), were introduced as explanatory variables. To maximize the relevant role of these explanatory variables, a step by step model was used. This model only introduced into the equation those variables with a significant contribution. Table 5 summarizes the coefficients obtained. Since some threats to normal distribution of SDM-9's items were found, a Mann-Whitney $U$ test was used for statistical contrasts. Table 3 summarizes the data obtained.

As can be observed, "type of medical care" is present in all the equations (and at the first step) except total score. There are two variables that play a significant role depending on the item/content - "age" contributes positively on the first items and "appointment type" (non-scheduled) on the latter items. "Sex" (women) only appears to play a role in predicting the "identification of preferences" item. Educational level only appears in the "follow-up" item. Overall score is only predicted (statistically significant) by "age".

Table 4 Percentage agreement with SDM-Q-9 contents in psychiatric outpatients and in primary care patients

\begin{tabular}{|c|c|c|c|c|}
\hline \multirow{2}{*}{$\begin{array}{l}\text { SDM process steps corresponding } \\
\text { to SDM-Q-9 Items }\end{array}$} & \multicolumn{2}{|c|}{ Psychiatric care } & \multicolumn{2}{|c|}{ Primary care } \\
\hline & Disagree & Agree & Disagree & Agree \\
\hline Disclosure that a decision needs to be made & 2.5 & 97.5 & 18.3 & 81.7 \\
\hline Formulation of equality of partners & 7.2 & 92.8 & 31.5 & 68.5 \\
\hline Equipoise statement & 51.1 & 48.9 & 20.7 & 79.3 \\
\hline Informing on the options' benefits and risks & 50.3 & 49.7 & 19.8 & 80.2 \\
\hline Investigation of patient's understanding and expectations & 8.4 & 91.6 & 8.7 & 91.3 \\
\hline Identification of preferences & 59.5 & 40.5 & 34.8 & 65.2 \\
\hline Negotiation & 53.8 & 46.2 & 32.2 & 67.8 \\
\hline Shared decision & 55.5 & 44.5 & 41.5 & 58.5 \\
\hline Arrangement of follow-up & 3.2 & 96.8 & 26.9 & 73.1 \\
\hline
\end{tabular}

Abbreviations: SDM, Shared Decision-Making; SDM-Q-9, nine-item Shared Decision-Making Questionnaire. 
Table 5 Regression analyses (step-by-step model) for content taking the following as explanatory variables: contextual clinical variables, socio-demographic variables, and type of medical care

\begin{tabular}{|c|c|c|}
\hline $\begin{array}{l}\text { SDM process steps } \\
\text { corresponding } \\
\text { to SDM-Q-9 items }\end{array}$ & $\begin{array}{l}\text { Variables into } \\
\text { the equation }\end{array}$ & $\beta(\mathrm{Cl})$ \\
\hline Disclosure that a decision & Medical care & $0.66(0.53 / 0.80)$ \\
\hline needs to be made & Age & $0.01(0.00 / 0.01)$ \\
\hline Formulation of equality & Medical care & $10.08(0.92 / 10.23)$ \\
\hline of partners & Age & $0.01(0.00 / 0.01)$ \\
\hline Equipoise statement & Medical care & $-0.70(-0.87 /-0.53)$ \\
\hline $\begin{array}{l}\text { Informing on the options' } \\
\text { benefits and risks }\end{array}$ & Medical care & $-0.71(-0.88 /-0.54)$ \\
\hline Investigation of patient's & Medical care & $-0.19(-0.32 /-0.06)$ \\
\hline $\begin{array}{l}\text { understanding and } \\
\text { expectations }\end{array}$ & Age & $0.01(0.00 / 0.01)$ \\
\hline Identification of & Medical care & $-0.62(-0.84 /-0.4 I)$ \\
\hline \multirow[t]{2}{*}{ preferences } & Appointment type & $-0.39(-0.64 /-0.15)$ \\
\hline & Sex & $0.21(0.02 / 0.040)$ \\
\hline \multirow[t]{2}{*}{ Negotiation } & Medical care & $-0.48(-0.69 /-0.27)$ \\
\hline & Appointment type & $-0.29(-0.52 /-0.05)$ \\
\hline \multirow[t]{2}{*}{ Shared decision } & Medical care & $-0.18(-0.25 /-0.11)$ \\
\hline & Appointment type & $-0.08(-0.16 /-0.01)$ \\
\hline Arrangement of & Medical care & $0.96(0.80 / 10.11)$ \\
\hline follow-up & Educational level & $-0.11(-0.19 /-0.03)$ \\
\hline Total score & Age & $0.09(0.01 / 0.17)$ \\
\hline
\end{tabular}

Abbreviations: SDM, Shared Decision-Making; SDM-Q-9, nine-item Shared Decision-Making Questionnaire; $\beta(\mathrm{Cl})$, Beta coefficients (confidence interval).

\section{Discussion}

The main aim of this study was to test whether there were differences in patients' perception of SDM according to the type of medical care setting (primary care versus psychiatric outpatient/specialized care). Considering SDM as a process integrated by several steps (from recognition that a decision needs to be made to final agreement on how to proceed), two very distinctive profiles were expected.

The increasing amount of scientific literature on SDM in the last decade focusing on patients with psychiatric disorders has revealed that psychiatric patients are generally interested in information and taking part in decision making, and that they often want more participation compared to what has been experienced. ${ }^{7,13,19-21}$ However, previous research in our community psychiatry health care setting has revealed that psychiatrists also show a favorable attitude towards involving patients in a process of reciprocal communication where patients' preferences, values, and expectations are considered, although they are more cautious in their attitude towards sharing decisions. ${ }^{14}$

Our results show no differences in final SDM between patients' perceptions, but when a contrast was made step by step in SDM (as items), several differences were found.
These differences support the fact that, regardless of the final results, the process to come to a shared decision has a distinctive profile, depending on the type of medical care. Psychiatric care emphasizes the initial steps, perhaps because there are already initial sufficient data for making a treatment decision. Primary care, however, emphasizes the final steps, perhaps because the time for a decision, according to the nature of consultation, is arriving.

Thus, psychiatric outpatients considered that despite their agreement at initial steps (when a decision must be made), they report their disagreement with the subsequent (successive) steps of the decision making process, including sharing decisions. On the other hand, most primary care patients reported having taken part in each step of the decision process.

These differences can also be observed when intermediate variables are considered. Regression analyses reveal that the type of medical care is the best predictor of the different steps in SDM. Sociodemographic variables (age, sex, and education) and clinical context variables (appointment type, treatment decision) play a different role according to each step. In general, sociodemographic variables (more agreement as age is increased) play a significant role in the initial steps, and a clinical variable (more agreement with scheduled appointments) plays a significant role in the subsequent steps. These results support the presence of different profiles in SDM according to medical care.

Variables such as sex or educational level do not play a significant role (with some minimal exceptions), and the absence of a significant role in treatment decision is noteworthy, since agreement is expected when there are no changes to treatment, and more difficulties in SDM when treatment is modified. Perhaps new considerations are required, including the patient's perception about a treatment change; patients can disagree about the need for a change in or continuation of treatment, according to how they perceive their health status.

At this point, a conceptual consideration may be made - the presence of differences at different steps of SDM according to type of medical care could represent a specific view of the concept of SDM. We measured SDM with a well validated questionnaire, the SDM-9-Q. The factorial validation of the original version in addition to the Spanish version of the questionnaire revealed a clear one-dimensional structure. ${ }^{17,18}$ This single factor solution of SDM-Q-9 can confound (and mask) the complex process of SDM and the independent role of each one of its steps, that, for us, are mostly represented by its items. The inter-item covariability 
cannot suppose, directly, that the items share the same conceptual framework. A single factor solution points out a similar pattern of responses to the items, but that implies that every item (or some items) can have independent conceptual identity by itself; we believe this is the case for SDM-Q-9. As we observed, it is necessary to analyze the different steps/ contents in SDM, because distinctive information patterns are present.

Our results showed that those patterns were quite different between primary care and psychiatric outpatient care settings - they reflect the different perspectives of the decision making process in each setting. The initial absence of differences in SDM-Q-9 general score between patients seen by psychiatrists and primary care patients coexisted with notable differences, according to different specific steps (item contents) of the SDM process. This indicates that the hierarchic process of SDM can be modified into an independent step by step procedure, which can account for the differences found in this study. Analyzing what those independent steps are could be a topic for further investigation.

Although we have information about the diagnosis of a considerable proportion of the patients involved in the study, we have not processed these data statistically because we considered these data unreliable. Patients' diagnoses were self-reported and some patients preferred not to report their diagnosis in order to maintain their anonymity and confidentiality. Moreover, the analysis of SDM according to psychiatric diagnosis is inherently complex because most diagnostic categories in psychiatry have not been shown to be valid; categories lack validity because they are not discrete entities with natural boundaries that separate them from other disorders. ${ }^{22}$ Furthermore, diagnostic systems such as the 4th edition of the Diagnostic and Statistical Manual of Mental Disorders (DSM-IV) ${ }^{23}$ and the International Classification of Diseases guidelines (ICD-10) ${ }^{24}$ foster diagnosing of comorbid conditions.

As a practical implication of these results, SDM occurs to a very limited extent in our routine medical outpatient setting in spite of the previous positive attitudes seen in our health care system. Psychiatrists and family physicians who are highly motivated to use SDM could improve their skills and benefit from SDM training programs where increased importance is placed on encouraging them to carry this concept into their professional activities. The different steps of SDM can now be viewed as specific independent phases that must be encouraged, according to different medical care settings. Finally, since patients perceive differences in SDM according to the different steps of the process, clinicians interested in SDM would need to pay attention to the SDM process as a whole.

Some limitations of this study should be considered. First and foremost, although the self-reporting (subjective) measures in the SDM process have been shown to be practical and low cost, have a low participant burden, and are well accepted, particularly when using the SDM-Q-9, self-report methods possess several limitations in terms of recall and response bias (eg, social desirability, inaccurate memory) and their inability to capture the absolute level of SDM. ${ }^{26}$

Another possible limitation is the representativeness of the samples used. These results are based on data drawn from consecutive samples of primary care patients and psychiatric outpatients. The fact that sampling was consecutive could mean that the samples studied may have a higher representation of high-frequency users of health care services and under-representation of low-frequency users. However, samples studied were obtained over a period of time several times longer than the waiting list, minimizing this potential bias. A challenge of any research survey is finding and recruiting participants from the target population. This study included suitable samples of patients with different backgrounds, which could have biased our results. In addition, nonparticipants were older than participants and included more men. In this sense, this sample may not be representative of the entire population attending psychiatric outpatient and primary health care settings. However, considering the high response rates achieved and the fact that the educational characteristics of the patients in this study correspond to those of the Spanish population, we may have mitigated the aforementioned limitation.

The SDM-Q-9 sub-item analysis carried out should be generalized and interpreted with some caution since the original questionnaire has been validated as a whole. Nevertheless, SDM-Q-9 is a theory-driven self-report questionnaire that defines the SDM process through theoretical key features and nine practical steps that may be seen as additive components rather than as a collection of homogeneous and equivalent items. ${ }^{16,17}$ However, sub-item analysis or comparison cannot directly lead to definitive conclusions, but rather it can generate a possible hypothesis since we would be using each item as a step in itself and perhaps other factors may be interrelated.

\section{Conclusion}

This study provides evidence that SDM is a complex process that needs to be analyzed according to its different steps. This process shows very different patterns in the primary care and 
psychiatric outpatient care settings, reflecting quite a different perspective of the decision making process.

\section{Authors' contributions}

CDC conceived of the idea and completed the data collection.

All authors participated in the design of the study and the analysis. This article has been read and revised by all authors. All authors read and approved the final manuscript.

\section{Disclosure}

The authors report no conflicts of interest in this work. This research was supported by Instituto de Salud Carlos III, FEDER Unión Europea (PI10/00955).

\section{References}

1. Deegan PE, Drake RE. Shared decision making and medication management in the recovery process. Psychiatr Serv. 2006;57(11): 1636-1639.

2. Hamann J, Leucht S, Kissling W. Shared decision making in psychiatry. Acta Psychiatr Scand. 2003;107(6):403-409.

3. Elwyn G, Edwards A, Kinnersley P, Grol R. Shared decision making and the concept of equipoise: the competences of involving patients in healthcare choices. Br J Gen Pract. 2000;50(460):892-899.

4. Härter M. Shared decision making - from the point of view of patients, physicians and health politics is set in place. Z Arztl Fortbild Qualitatssich. 2004;98(2):89-92. German.

5. Elwyn G, Charles C. Shared Decision Making: From Conceptual Models to Implementation in Clinical Practice. In: Shared DecisionMaking in Health Care: Achieving evidence-based patient choice. Edwards A, Elwyn G, editors. Oxford University press: New York; 2009:117-121.

6. Fenton WS. Shared decision making: a model for the physician-patient relationship in the 21st century? Acta Psychiatr Scand. 2003;107(6): 401-402.

7. Patel SR, Bakken S, Ruland C. Recent advances in shared decision making for mental health. Curr Opin Psychiatry. 2008;21(6):606-612.

8. Simon D, Wills CE, Härter M. Shared decision-making in mental health. In: Shared Decision-Making in Health Care: Achieving evidence-based patient choice. Edwards A, Elwyn G, editors. Oxford University press: New York; 2009:269-276.

9. Drake RE, Deegan PE, Rapp C. The promise of shared decision making in mental health. Psychiatr Rehabil J. 2010;34(1):7-13.

10. Perestelo-Pérez L, González-Lorenzo M, Rivero-Santana AJ, Pérez-Ramos J. Herramientas de ayudapara la toma de decisiones de los pacientes con depresión. Plan de Calidad para el SNS del MSPS. SESCS; 2010. Informes de ETS: SESCS N² 2007/04.

11. Perestelo-Perez L, Gonzalez-Lorenzo M, Perez-Ramos J, RiveroSantana A, Serrano-Aguilar P. Patient involvement and shared decision-making in mental health care. Curr Clin Pharmacol. 2011;6(2): $83-90$.

Neuropsychiatric Disease and Treatment

\section{Publish your work in this journal}

Neuropsychiatric Disease and Treatment is an international, peerreviewed journal of clinical therapeutics and pharmacology focusing on concise rapid reporting of clinical or pre-clinical studies on a range of neuropsychiatric and neurological disorders. This journal is indexed on PubMed Central, the 'PsycINFO' database and CAS.
12. Duncan E, Best C, Hagen S. Shared decision making interventions for people with mental health conditions. Cochrane Database Syst Rev. 2010;(1):CD007297.

13. De las Cuevas C, Rivero-Santana A, Perestelo-Pérez L, Pérez-Ramos J, Serrano-Aguilar P. Attitudes toward concordance in psychiatry: a comparative, cross-sectional study of psychiatric patients and mental health professionals. BMC Psychiatry. 2012;12:53.

14. De las Cuevas C, Rivero A, Perestelo-Perez L, Gonzalez M, Perez J, Peñate W. Psychiatric patients' attitudes towards concordance and shared decision making. Patient Educ Couns. 2011;85(3): e245-e250.

15. de Las Cuevas C, Rivero-Santana A, Perestelo-Perez L, et al. Mental health professionals' attitudes to partnership in medicine taking: a validation study of the Leeds Attitude to Concordance Scale II. Pharmacoepidemiol Drug Saf. 2012;21(2):123-129.

16. Simon D, Schorr G, Wirtz M, et al. Development and first validation of the shared decision-making questionnaire (SDM-Q). Patient Educ Couns. 2006;63(3):319-327.

17. Kriston L, Scholl I, Hölzel L, Simon D, Loh A, Härter M. The 9-item Shared Decision Making Questionnaire (SDM-Q-9). Development and psychometric properties in a primary care sample. Patient Educ Couns. 2010;80(1):94-99.

18. De las Cuevas C, Perestelo-Perez L, Rivero-Santana A, Cebolla-Martí A, Scholl I, Härter M. Validation of the Spanish version of the 9-item Shared Decision Making Questionnaire. Health Expect. 2013 (in press).

19. Hamann J, Cohen R, Leucht S, Busch R, Kissling W. Do patients with schizophrenia wish to be involved in decisions about their medical treatment? Am J Psychiatry. 2005;162(12):2382-2384.

20. Adams JR, Drake RE, Wolford GL. Shared decision-making preferences of people with severe mental illness. Psychiatr Serv. 2007;58(9):1219-1221.

21. O'Neal EL, Adams JR, McHugo GJ, Van Citters AD, Drake RE, Bartels SJ. Preferences of older and younger adults with serious mental illness for involvement in decision-making in medical and psychiatric settings. Am J Geriatr Psychiatry. 2008;16(10):826-833.

22. Kendell R, Jablensky A. Distinguishing between the validity and utility of psychiatric diagnoses. Am J Psychiatry. 2003;160(1):4-12.

23. Patel SR, Bakken S. Preferences for participation in decision making among ethnically diverse patients with anxiety and depression. Community Ment Health J. 2010;46(5):466-473.

24. American Psychiatric Association. Diagnostic and Statistical Manual of Mental Disorders, 4th ed. Washington: American Psychiatric Association; 1994.

25. World Health Organization. The ICD-10 Classification of Mental and Behavioural Disorders. Clinical descriptions and diagnostic guidelines. Geneva: World Health Organization; 1992. Available from: http://www.who.int/classifications/icd/en/bluebook.pdf. Accessed September 1, 2012.

26. Nevin RL. Low validity of self-report in identifying recent mental health diagnosis among US service members completing Pre-Deployment Health Assessment (PreDHA) and deployed to Afghanistan, 2007: a retrospective cohort study. BMC Public Health. 2009;9:376.

27. De las Cuevas, Castresana C, González de Rivera JL. Self-reports and Response Biases. AnPsiquiatría. 1992;8:362-366.

The manuscript management system is completely online and includes a very quick and fair peer-review system, which is all easy to use. Visit http://www.dovepress.com/testimonials.php to read real quotes from published authors. 